\title{
Materials for reliable interocclusal measurements
}

\section{Clinical factors and clinical variation influencing the reproducibility of interocclusal recording methods \\ A. Eriksson, G. Öckert-Eriksson, P. Lockowandt and O. Eriksson Br Dent J 2002; 192: 395-400}

\section{Objective}

The reproducibility of clinical records of the occlusion was assessed in three dimensions using mounted casts. Three distinct areas were examined: 1) mandibular positions (intercuspal position (IP) or retruded contact position (RCP)), 2) materials used in recording the occlusion, 3 ) clinical variation.

\section{Design}

Interocclusal records were made in a random order of three patients: one fixed prosthodontics case, one removable partial denture case and one complete denture case, with two different types of waxes, record rims, two different brands of vinyl polysiloxanes and one irreversible hydrocolloid.

\section{Setting and Subjects}

Private practice and Karolinska Institute, Huddinge, Sweden. One general dental practitioner and three voluntary patients.

\section{Results}

Point estimation of variance components indicate that 70-93\% of the variation of the positions of the mounted casts are caused by: 1) clinical variation for all three cases and in three directions, 2) the influence of recording materials 0-29\%, and 3) mandibular positions (IP/RCP) $0-11 \%$. The ranges of the positions of the mounted casts were lower for the dentate case $(0.04-1.39 \mathrm{~mm})$ than for the partially dentate case $(0.17-2.65 \mathrm{~mm})$, which in turn was lower than those for the edentulous case (1.42-5.59 $\mathrm{mm}$ ).

\section{Conclusion}

Clinical variation seems to dominate the variation in positions of mounting casts when making interocclusal records, rather than mandibular position or the recording materials used. Therefore a dentist who makes one single interocclusal record cannot presume that it will reproduce the interocclusal relationship intended, which in the present study was most obvious for the edentulous case. The results showed that impression materials stabilised by a tray did not differ significantly from waxes and record rims concerning the reproducibility. Therefore the stabilised impression materials are an alternative, which also give additional advantages like reduction of appointments as well as superior accuracy.

\section{IN BRIEF}

- A new alternative interocclusal recording method using impression material reinforced by a stabilising tray has been compared to conventional methods in three clinical cases.

- There were no significant differences between the methods because clinical factors and clinical variation rather than recording materials or mandibular positions determined the range of the positions of the mounted casts.

- The new interocclusal recording method ie a combination of impression material/stabilising tray in most cases can replace record rims and the number of appointments therefore can be reduced.

\section{COMMENT}

Accurate interocclusal registrations are an integral part of restorative dentistry. Previous studies have shown that there is significant inter-operator variability in recording interocclusal registrations. The aim of this study was to examine the three dimensional reproducibility of mounting casts by a single clinician, using a variety of recording materials.

Three patients, dentate, partially dentate and edentulous respectively participated in the study. Interocclusal records were taken for the three patients using two types of waxes, two brands of polyvinylsiloxane and a hydrocolloid.

The results of the study demonstrated that clinical variation seemed to dominate the variation in the positions of mounting casts when making records, as opposed to the recording materials used or the mandibular position. Unsurprisingly the variation was greatest for the edentulous patient and was least for the dentate patient. There was no significant difference in reproducibility of the recording methods. The authors therefore recommended the use of the least time consuming method which they themselves had developed.

The study adds to our knowledge and highlights the difficulty associated with this important phase of restorative dentistry. It is of interest to the clinician that all of the recording methods gave comparable results. It is a matter of considerable interest that an experienced dentist was unable to achieve consistent recordings for any of the patients with any of the methods.

Dr Daryll Jagger

Senior Lecturer in Restorative Dentistry

Bristol Dental Hospital and School 